\title{
Théorie informationnelle du schéma
}

\section{Abraham Moles}

\section{(2) OpenEdition}

Journals

Édition électronique

URL : https://journals.openedition.org/rbnu/1688

DOI : $10.4000 /$ rbnu. 1688

ISSN : 2679-6104

\section{Éditeur}

Bibliothèque nationale et universitaire de Strasbourg

\section{Édition imprimée}

Date de publication : 1 novembre 2014

Pagination : 88-91

ISBN : 9782859230548

ISSN : 2109-2761

\section{Référence électronique}

Abraham Moles, "Théorie informationnelle du schéma », La Revue de la BNU [En ligne], 10 | 2014, mis en ligne le 01 novembre 2014, consulté le 17 mai 2021. URL : http://journals.openedition.org/rbnu/ 1688 ; DOI : https://doi.org/10.4000/rbnu.1688

\section{(C) $10(0$}

La Revue de la BNU est mise à disposition selon les termes de la Licence Creative Commons Attribution - Pas d'Utilisation Commerciale - Partage dans les Mêmes Conditions 4.0 International. 

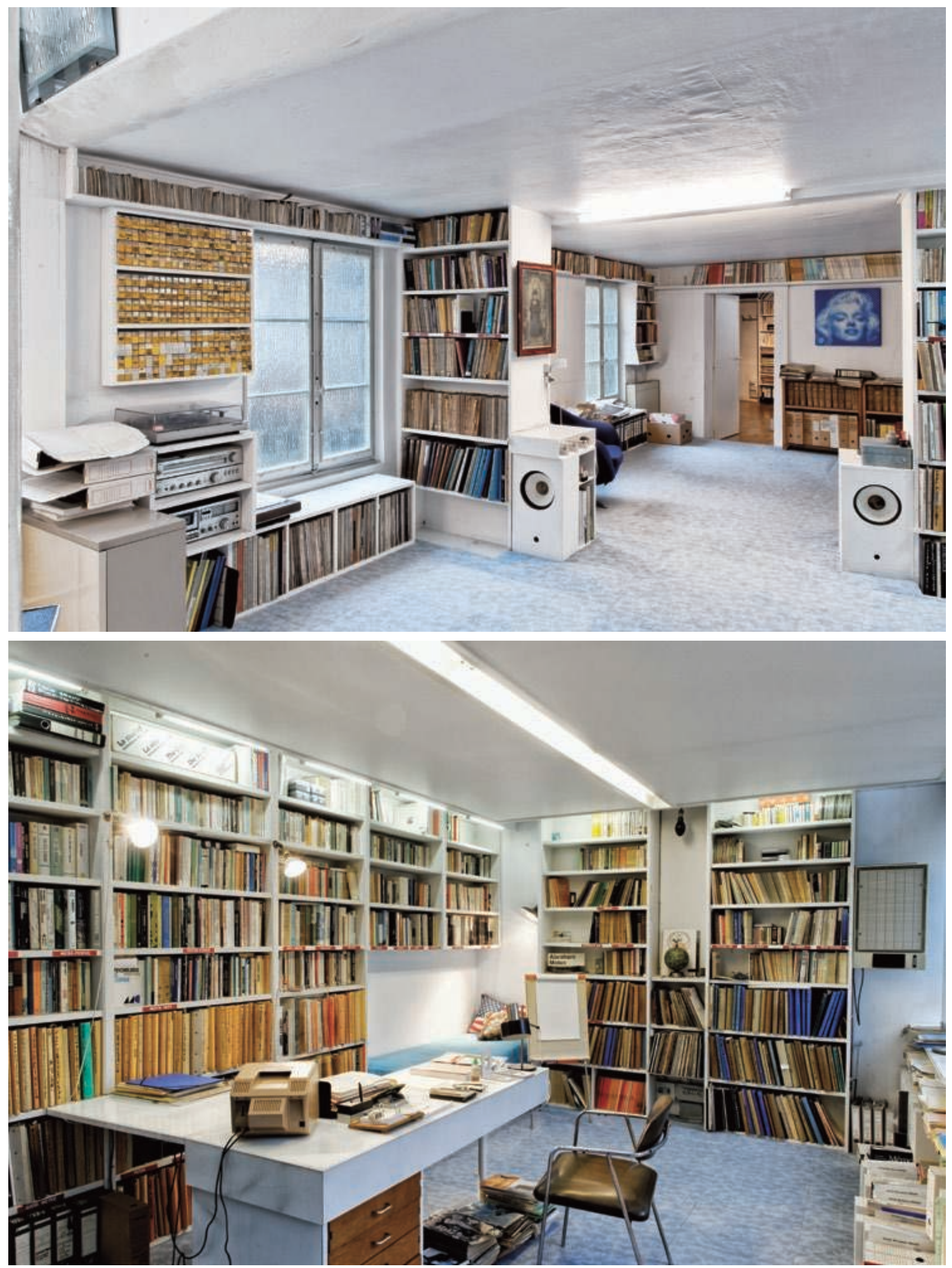

Vue du lieu de travail d'Abraham Moles et de sa bibliothèque (Strasbourg, 2013) 


\section{THÉORIE INFORMATIONNELLE DU SCHÉMA}

\section{LA BIBLIOTHÈQUE D'ABRAHAM MOLES}

Précurseur des études en sciences de l'information et de la communication, ingénieur de formation (électricité et acoustique), diplômé de l'Université de Grenoble, docteur ès sciences (Sorbonne, 1952) et ès lettres (Sorbonne, 1954), Abraham Moles (1920-1992) a enseigné à l'Université de Strasbourg dès 1966. Il y a créé l'Institut de psychologie sociale (appelé aussi l'" École de Strasbourg "), qu'il dirigea jusqu'en 1987. Plusieurs de ses anciens élèves, aujourd'hui universitaires, se retrouvent au sein de l'Association internationale de micropsychologie et de psychologie sociale des communications.

En 2013, Élisabeth Rohmer-Moles, sa veuve, a proposé de faire don de la bibliothèque d'Abraham Moles à la BNU. Remarquable à plus d'un titre, elle comprend, outre les ouvrages qu'il a acquis ou reçus, les livres et les articles qu'il a écrits, ainsi que de nombreux documents liés directement à ses activités de recherche et d'enseignement. L'ensemble représente environ 7 ooo volumes (monographies, revues et dossiers).

Il y a une vingtaine d'années, un groupe de chercheurs et d'universitaires, membres de l'Association internationale de micropsychologie, avait travaillé avec un conservateur de la BNU. Il s'agissait alors de collecter et de recenser les articles d'Abraham Moles. En 1996, 46 microfiches avaient ainsi été éditées par la BNU, reproduisant 326 articles, soit environ un tiers du total des articles écrits par A. Moles.

L'entrée de sa bibliothèque dans les collections de la BNU s'inscrit dans la continuité directe de ce travail. Elle permettra l'accès aux sources et aux travaux prépa- ratoires, aux annotations et aux commentaires du chercheur. Elle permettra aussi, d'un point de vue bibliothéconomique, d'étudier l'agencement des documents et leur classement, révélateurs de la manière dont il organisait les domaines du savoir dans son environnement de travail. Il s'agit pour la BNU de conserver la spécificité de ce "dispositif de savoir " qui pourrait, avec bien d'autres facettes de ce formidable ensemble, faire l'objet de travaux d'étude et de recherche.

Formés simultanément aux sciences dites "exactes " et aux sciences dites "humaines", ayant reçu l'apport de la pensée cartésienne et positiviste de la culture française et les influences diverses de la pensée d'Europe centrale et de l'empirisme anglo-saxon, nous avons, au cours d'une carrière qui n'a pris son plein développement qu'à l'université, essayé de composer ces influences pour le bénéfice des sciences sociales, celles qu'on appelle ailleurs les "sciences de l'esprit", dans lesquelles nous voyons un nouveau dynamisme de la société, comparable à ce que fut l'essor technique des sciences de la nature dans les décennies de notre jeunesse (Abraham Moles et Élisabeth Rohmer, Le cursus scientifique d'Abraham Moles).

Le choix d'un texte inédit d'Abraham Moles dans le domaine des sciences, domaine auquel est consacré le présent numéro de La Revue de la BNU, a été difficile : fallait-il rechercher un texte dans la variété des champs scientifiques dans lesquels Moles a travaillé, allant ainsi du domaine des sciences dites dures à celui plus récent des communications, ou encore à celui des "sciences de l'imprécis " ? De nombreux textes, et même brevets, étaient disponibles, en particulier dans le domaine de l'acoustique. Un titre a priori humoristique comme celui d'un texte paru il y a fort longtemps dans une 
revue scientifique (Pourquoi deux violons font-ils plus de bruit qu'un seul ?) était tentant. De même de nombreux textes conduisant à l'élaboration des musiques concrètes ou d'autres davantage liés à la physique des matériaux.

Finalement, considérant que de nombreuses sciences et techniques se servaient du schéma, que la réflexion portant sur cet aspect est restée relativement peu développée, nous avons décidé de proposer un texte ancien, datant des années 70, mais dont l'actualité reste évidente. L'émergence de l'informatique, son utilisation dans le champ graphique, ont certes permis le développement des schémas dans de nombreux domaines, donnant lieu à d'innombrables notices, mais les règles d'intelligibilité dans leur réalisation, telles que décrites dans cet article, n'ont guère varié. Concluons ici en citant Valéry, luimême souvent cité par Moles : "Plus un esprit est pratique, plus il est abstrait ".

\section{Dominique Grentzinger, Elisabeth Rohmer-Moles}

\section{THÉORIE INFORMATIONNELLE DU SCHÉMA}

Schéma, schématisme, schématisation sont parmi les termes les plus fréquemment rencontrés dans le vocabulaire de l'activité créatrice. Le concept de schéma, défini comme une représentation simple et abstraite d'un phénomène ou d'un objet du monde extérieur, paraît prendre une importance de plus en plus grande au fur et à mesure que notre civilisation tend à être envahie par la pensée mécanique qui consomme et produit des schémas, et par l'inflation sémantique de ce qui, de près ou de loin, se rattache à une logique quelconque, à un logos normalisé. Le schéma est le lieu géométrique du contact entre l'homme et l'ordinateur : les efforts les plus récents dans le domaine de l'interface homme/machine, en particulier les systèmes de " graphic display ", convergent tous vers la possibilité de faire lire un schéma par une machine et de ramener l'effort du programmeur à l'établissement d'un bon schéma.

Les schémas sont des systèmes de pensée universels, normalisés, qui existent à tous les niveaux, depuis l'illustration d'un livre technique jusqu'aux représentations vectorielles les plus abstraites ; ils constituent un outil permanent de l'esprit humain dans l'appréhension du réel. On a pu dire que penser c'était schématiser, et on peut effectivement se demander si l'intelligence n'est pas synonyme de l'aptitude à faire des schémas. Il semble pourtant qu'il existe d'autres mécanismes de la pensée, mais la pensée schématique paraît liée à la catégorie psychologique de ceux qui pensent en projetant dans l'espace, par rapport à ceux qui pensent en abstractions pures.

Le schéma s'introduit dans la vie quotidienne de la pensée, sous des formes humbles et multiples. Il se propose comme une abréviation ("Un bon schéma vaut mieux qu'un long discours "), comme une simplification et comme une recodification abstraite de l'univers. Le terme d'intelligence sera pris comme synonyme de l'aptitude à faire des schémas, dans la mesure où la compréhension peut se ramener elle-même à une réduction de combinaisons de connaissances, mais il n'est pas certain que la totalité du monde des sens soit réductible à son contenu conceptuel.

Il existe d'innombrables types de schémas : le schéma de l'installation électrique utilisé par l'artisan qui gribouille sur une feuille de papier quelques traits et quelques symboles; le schéma, fait par le géologue, des interactions entre espèces aux différents âges de la Terre ; l'organigramme qui définit la structure d'une entreprise ; la description anatomique qui met en place les muscles du cou ; le panneau routier qui explique à l'automobiliste une déviation ; l'organigramme préparatoire au programme d'une machine à calculer ; la courbe compliquée tracée par le vecteur de l'activité cardiaque dans un espace de représentation. Tous ces éléments entrent dans la dénomination générale de "schéma " : ils présentent des degrés divers d'abstraction, de normalisation, de fini.

\section{La situation schématique}

Comment décrire de façon adéquate le monde des schémas ? C'est le problème de la dimensionnalisation du système schématique : existe-t-il des grandeurs caractéristiques, de caractère continu, indépendantes les unes des autres, qui permettent de décrire tout l'ensemble des schémas ? Le schéma est le produit d'une communication, comportant un émetteur qui crée le schéma, un récepteur qui le lit et le comprend, ceux-ci se trouvant réunis par l'utilisation commune d'un même répertoire d'éléments, signes ou symboles, déjà connus a priori, répertoriables et combinables entre eux selon certaines 
règles. Le schéma apparaît donc comme l'un des cas particuliers des mécanismes de communication et, par là, suggère un parallélisme avec un langage dont il possède toutes les caractéristiques : des signes, un vocabulaire, une syntaxe, une logique, une intelligibilité.

C'est de la prise de conscience de cette situation que partira le sémiologue qui cherchera à définir l'ensemble de ces aspects pour construire une théorie informationnelle du schéma. L'électronicien, qui lit le schéma d'un récepteur de télévision qui lui a été fourni par le constructeur, effectue une opération linguistique : il dégage les éléments, en maîtrise les combinaisons, et appréhende une signification, les transformations du signal aux différents niveaux de la structure que lui décrit le schéma.

\section{La dimension d'iconicité}

Il s'agit donc de représenter, mais en même temps d'abstraire et de réduire la réalité du monde à des signes intelligibles, découper les contours, dégager les universaux. L'acte même de donner des noms, de leur attribuer des symboles ou des numéros, sont les opérations du schématisme. Mais l'image même donnée d'un objet " décolle " de cet objet une enveloppe de représentation pour la projeter sur une feuille, et cette image est déjà un opérateur sur lequel on peut agir. On réduira dans une même perspective la variété de ces aspects de la schématisation à partir des concepts réciproques d'abstraction ou d'iconicité. L'iconicité, grandeur opposée à l'abstraction, serait, pour ainsi dire, la quantité de réalisme, la vertu iconale, la quantité d'imagerie immédiate, contenue ou retenue dans le schéma. C'est la proportion de concret conservée dans le schéma. L'objet tel qu'il est posséderait une iconicité totale, le mot qui le désigne (" le mot chien ne mord pas ", disait de Saussure) une iconicité nulle : telles sont les deux extrémités de l'échelle.

Le schéma électronique, l'organigramme sont typiques du langage sémantique et possèdent une grammaire relativement développée dans laquelle le message, c'est-à-dire l'assemblage d'éléments, est infiniment plus important que l'alphabet des signes : les signes sont ici normalisés à l'échelle internationale et sont assemblés selon les lois d'une syntaxe et d'une esthétique. Il faut également séparer les schémas proprement dits des représentations graphiques, à deux ou trois dimensions, que nous proposent les systèmes de coordonnées. Les graphiques cartésiens en $\mathrm{O}, \mathrm{x}, \mathrm{y}$, les diagrammes économiques, les lignes de courant d'écoulement en hydrodynamique, les lignes équipotentielles d'un champ dynamique, ne paraissent pas entrer dans la catégorie des schémas : ils représentent le réel dans des espaces abstraits ; ils ne prétendent pas, au moins principalement, à schématiser, ils visent à l'exactitude et non à la norme, au réalisme et non à l'abstraction. En revanche, les pictogrammes, quelquefois basés eux-mêmes sur des représentations graphiques, entrent dans la catégorie des schémas, puisqu'ils modifient par l'abstraction ou le symbole les relations de l'individu avec le réel.

\section{La dimension de complexité}

Le fait que le schéma soit un message constitué d'éléments généralement symboliques, assemblés dans un certain ordre, conduit naturellement à une notion générale, directement dérivée de la théorie de l'information, celle de complexité ou de taux d'information du schéma ainsi réalisé, qui se trouve corrélative, en principe, de la complexité de l'objet ou du phénomène représenté. En fait, il s'agit là d'une autre dimension de l'univers des schémas, orthogonale à l'idée d'iconicité, et qui permet de réordonner celle-ci en fonction de cette complexité. Ainsi que l'avait bien décrit Shannon, la complexité d'un système est liée à deux facteurs distincts : d'une part, le nombre des éléments utilisés dans le système (longueur du message) ; d'autre part, l'originalité des associations de ceux-ci entre eux : un schéma comportant peu d'éléments peut être relativement complexe ; un schéma comportant un grand nombre d'éléments alignés de façon routinière ou répétitive, appréhensibles dans un seul regard par le spectateur, peut être, en fait, moins complexe. L'utilisation des règles de grammaire et de syntaxe a pour effet d'augmenter la redondance de l'assemblage, donc de la prégnance du schéma, en d'autres termes de son intelligibilité. Pratiquement, intelligibilité et complexité d'un schéma varieront en sens inverse, mais sont des valeurs co-extensives, corrélées négativement, en fait, antinomiques.

En résumé, nous avons fait émerger deux grandeurs fondamentales de l'univers des schémas :

1 - leur degré d'iconicité ou, réciproquement, d'abstraction ;

2- leur degré de complexité ou, réciproquement, d'intelligibilité. 


\section{Les dimensions complémentaires de l'univers des schémas}

D’autres dimensions jouent pourtant dans la perception schématique, entre autres :

- la grandeur apparente : encombrement du champ visuel par le message dans les conditions normales d'utilisation ;

— la dimension esthétique : un schéma peut être fonctionnellement et logiquement adéquat, tout en restant terne et peu esthétique. En général, une fois les conditions générales déterminées, le dessinateur dispose d'un champ de liberté dont il fera usage à son gré ;

- un caractère important dans les applications est le concept de distance sémantique entre l'image et le texte qu'elle accompagne. Cette idée de distance sémantique, ou de pertinence de la figure par rapport au texte, serait déterminante dans l'illustration d'ouvrages scientifiques ou techniques. Son existence n'implique d'ailleurs pas nécessairement que cette distance soit faible, c'est-à-dire que la figure soit totalement pertinente par rapport au texte qu'elle accompagne, même si c'est le cas le plus fréquent.

- enfin, un dernier caractère important dans l'utilisation sociale du schéma est ce qu'on peut appeler son taux de normalisation, c'est-à-dire l'écart plus ou moins grand entre la forme de réalisation du schéma, ainsi que le choix des éléments symboliques qu'il comprend, et une norme idéale, en tout cas internationale, souvent traduite dans des textes réglementaires (AFNOR, DIN, ICOGRADA, etc.). Le degré d'écart, statistiquement exprimé entre le message réel et une norme, c'est le respect d'une grammaire ou d'une syntaxe internationales, c'est un taux d'universalité qui n'est corrélé que faiblement à l'intelligibilité, mais qui représente une autre caractéristique - fortement sociale celle-là du rôle de la schématisation : l'ensemble des " dispersions » des symboles nationaux du Code de la route, par rapport à une norme internationale, généralement de caractère topologique, est un critère de schématisation et de l'emploi pratique de celle-ci.

\section{La création schématique}

Dans la réalisation des schémas, on distinguera en fait cinq étapes qui fournissent une bonne représentation des étapes même de la créativité artistique :

1 -Étape heuristique : l'individu transmetteur trouve une idée de schématisation, conçoit un problème sous forme schématique, reliant dans son esprit une série d'éléments simples par une suite de liaisons dynamiques dans un "espace de représentation ". En fait, c'est généralement à ce niveau que se situe la compréhension et que se justifie éventuellement la formule : comprendre, c'est schématiser. L'électronicien qui gribouille un schéma pour comprendre un problème utilise une des méthodes heuristiques fondamentales de l'esprit.

2-Pour aller plus loin, la normalisation et la correction grammaticale interviennent : les symboles sont normalisés, ramenés à ceux qui sont acceptables par tout récepteur, en tout cas sur le plan topologique ; les liaisons sont revues et corrigées : citons par exemple le " retour à la masse " ou le point " haute tension " des anodes, l'insertion des générateurs d'énergie dans les schémas électroniques, etc.

3-L'étape suivante est la réalisation proprement dite du brouillon à l'état définitif : elle se fait à l'atelier de projet, et fait intervenir les éléments normalisateurs pour établir une géométrie des parallèles, des orthogonales et des contrastes. A ce stade, des aspects topologiques, tels que la disposition des éléments à relier avec le minimum de croisements, viennent exercer une action régulatrice sur l'esprit créateur.

4-Au quatrième stade, intervient le développement esthétique où l'on cherche, sans modifier structure topologique et norme, à améliorer l'aspect esthétique du schéma.

5-Le dernier stade est celui du contrôle scientifique, de la comparaison, de l'épreuve de lisibilité et d'acceptation, de l'estimation de la pertinence et de l'adéquation du schéma à son environnement ou aux messages plus globaux auxquels il est incorporé. La parfaite lisibilité reste une condition essentielle pour tout schéma.

\section{Abraham Moles}


Nous donnons ci-dessous, à la suite des travaux effectués à notre séminaire à la Hochschule für Gestaltung à Ulm, une échelle d'iconicité en 12 points : précisant les différents degrés essentiels de cette abstraction progressive, de ce dépouillement des qualités sensibles de l'objet ou du phénomène, en prélevant tout d'abord les exemples dans I'univers géométrique, et laissant provisoirement de cóté les schémas temporels, dont le théâtre ou le cinéma offrent des exemples assez importants pour ètre étudiés séparément : "le théátre est un schéma", disait déjà Bergson.

\begin{tabular}{|c|c|c|c|}
\hline & \multicolumn{3}{|c|}{ ECHELLE D'ICONICITE DECROISSANTE } \\
\hline$N^{\circ}$ & DEFINITION & CRITERE & EXEMPLES VARIES \\
\hline 0 & L'objet lui-mème. & $\begin{array}{l}\text { Mise eventuelle entre pa- } \\
\text { renthèses au sens de Husserl. }\end{array}$ & $\begin{array}{l}\text { La vitrine de magasin, l'expo. } \\
\text { sition. Le thème du langage } \\
\text { naturel de Swiftb Laputa. }\end{array}$ \\
\hline 1 & $\begin{array}{l}\text { Modèle bi ou tridimensionnel } \\
\text { à l'échelle. }\end{array}$ & $\begin{array}{l}\text { Couleurs et matériaux arbi- } \\
\text { traires. }\end{array}$ & Etalages factices. \\
\hline 2 & $\begin{array}{l}\text { Schéma bi ou tridimensionnel } \\
\text { réduit ou augmenté. Repré- } \\
\text { sentation anamorphosée. }\end{array}$ & $\begin{array}{l}\text { Couleurs ou matériaux choi- } \\
\text { sis selon des critetres logiques. }\end{array}$ & $\begin{array}{l}\text { Cartes à } 3 \text { dimensions : globe } \\
\text { terrestre, carte géologique. }\end{array}$ \\
\hline 3 & $\begin{array}{l}\text { La photographie ou projection } \\
\text { réaliste sur un plan. }\end{array}$ & $\begin{array}{l}\text { Projection perspective rigou. } \\
\text { reuse, demi-teintes, ombres. }\end{array}$ & Catalogues illustrés, affiches: \\
\hline 4 & $\begin{array}{l}\text { Dessin ou photographie dits } \\
\text { "détournés" (opération visualle } \\
\text { de l"universal aristotélicien). } \\
\text { Profils en dessin. }\end{array}$ & $\begin{array}{l}\text { Critères de continuité du } \\
\text { contour et de fermeture de } \\
\text { la forme. }\end{array}$ & $\begin{array}{l}\text { Affiches, catalogues, prospeo- } \\
\text { tus. }\end{array}$ \\
\hline 5 & $\begin{array}{l}\text { Schéma anatomique ou de } \\
\text { construction. }\end{array}$ & $\begin{array}{l}\text { Ouverture du carter ou de } \\
\text { l'envaloppe. Respect de la } \\
\text { topographie, Arbitraire des } \\
\text { valeurs, quantification des } \\
\text { éléments et simplification. }\end{array}$ & $\begin{array}{l}\text { Coupe anatomique - Coupe } \\
\text { d'un moteur à explosion. } \\
\text { Plan de cliblage d'un récep- } \\
\text { reur de radio. Carte gégra- } \\
\text { phique }\end{array}$ \\
\hline 6 & Vue "éclatée": & $\begin{array}{l}\text { Disposition perspective des } \\
\text { piêces selon leurs relations } \\
\text { de voisinage topologique. }\end{array}$ & 4 \\
\hline 7 & $\begin{array}{l}\text { Schéma de principe (électri- } \\
\text { cíté et électronique). }\end{array}$ & $\begin{array}{l}\text { Remplacement des b́léments } \\
\text { par des symboles normalisés. } \\
\text { Passage de la topographie a } \\
\text { la topologie. Géométrisation. }\end{array}$ & $\begin{array}{l}\text { Plan schématisè du métro de } \\
\text { Londres. Plan de cablage d'un } \\
\text { récepteur de TV ou d'une par- } \\
\text { tie de radar. Schéma unifilaire } \\
\text { en électrotechnique. }\end{array}$ \\
\hline 8 & Organigramme ou Block schéma & $\begin{array}{l}\text { Les eléments sont des boites } \\
\text { noires fonctionnelles, reliées } \\
\text { par des connections logiques } \\
\text { analyse des fonctions logiques: }\end{array}$ & $\begin{array}{l}\text { Organigramme d'une entrepeise } \\
\text { Flow chart d'un programme } \\
\text { d'ordinateur. Série d'opéra- } \\
\text { tions chimiques }\end{array}$ \\
\hline 9 & Schéma de formulation. & $\begin{array}{l}\text { Relation logique et non topo- } \\
\text { logique dans un espece non } \\
\text { géométrique entre éléments } \\
\text { abstraits. Les liaisons sont } \\
\text { symboliques, tous les dléments } \\
\text { sont visibles. }\end{array}$ & $\begin{array}{l}\text { Formules chimiques dévelop. } \\
\text { pées. Sociogrammes. }\end{array}$ \\
\hline 10 & Schéma en espaces complexes & $\begin{array}{l}\text { Combinaison dans un méme } \\
\text { espace de représentation } \\
\text { dëléments schématiques } \\
\text { (flèche, droite, plan, objet) } \\
\text { appartenant à des systèmes } \\
\text { différents. }\end{array}$ & $\begin{array}{l}\text { Forces et positions géomètri- } \\
\text { ques sur une structure métal- } \\
\text { lique : schémas de statique } \\
\text { graphique, polvgone de } \\
\text { Crémona. }\end{array}$ \\
\hline 11 & $\begin{array}{l}\text { Schéma en espace purement } \\
\text { atsstrait et schéma vectoriel. }\end{array}$ & $\begin{array}{l}\text { Representation graphique } \\
\text { dans un espace métrique } \\
\text { abstrait, de relations entre } \\
\text { grandeurs vectorielles. }\end{array}$ & $\begin{array}{l}\text { Graphiques vectoriels en } \\
\text { électro-technique. Triangle } \\
\text { de Kapp. Polygone de Blondel } \\
\text { pour un moteur asynchrone. } \\
\text { Diagramme de Maxwell. Ob- } \\
\text { jets sonores, triangle des } \\
\text { voyelles. }\end{array}$ \\
\hline 12 & $\begin{array}{l}\text { Description en mots norma- } \\
\text { lisis ou en formules algébriques. }\end{array}$ & $\begin{array}{l}\text { Signes purement abstraits } \\
\text { sans connexion imaginable } \\
\text { avec le signifie. }\end{array}$ & Equations et formules. Textes. \\
\hline
\end{tabular}

Fiche de travail ayant servi à A. Moles pour la rédaction de son article (provenance inconnue, fonds Moles-BNU) 